\title{
Baseline characteristics, management and long-term outcomes of different etiologies of cardiac tamponade evaluated in a cohort of $\mathbf{3 4 0}$ patients
}

\author{
Mária Adamczyk ${ }^{1}$, Jarosław Wasilewski ${ }^{1}$, Jacek T. Niedziela ${ }^{1}$, Michał O. Zembala², Mariusz Gąsior ${ }^{1}$ \\ ${ }_{1}^{1}{ }^{\text {rd }}$ Department of Cardiology, Faculty of Medical Sciences in Zabrze, Medical University of Silesia in Katowice, Silesian Centre \\ for Heart Diseases, Zabrze, Poland \\ 2Department of Cardiac, Vascular and Endovascular Surgery and Transplantation, Silesian Center for Heart Diseases, \\ School of Medicine with the Division of Dentistry in Zabrze, Medical University of Silesia in Katowice, Poland
}

Kardiochirurgia i Torakochirurgia Polska 2021; 18 (4): 216-220

\begin{abstract}
Introduction: Studies on the etiology of cardiac tamponade (CT) are scarce or lacking follow-up, and usually include small or highly selected groups of patients.

Aim: To evaluate the various etiologies and outcomes of CT in a cohort of patients treated in a tertiary care hospital encompassing cardiology, cardiac surgery and intensive care units.

Material and methods: We retrospectively analyzed all adult patients hospitalized in the Silesian Centre for Heart Diseases in Zabrze (Poland) between January 2008 and December 2018, who required therapeutic pericardiocentesis or pericardiotomy due to $\mathrm{CT}$. All various etiologies of CT were presented and assigned to the main etiology groups. For each group basic characteristics, in-hospital management, in-hospital and up to 2-year mortality were analyzed.

Result: Among 340 patients with CT, 56\% were men. The leading etiology groups included patients after invasive cardiac procedures, patients following postpericardiotomy (PCT) syndrome and the patients with neoplasm. Patients with end stage renal failure, PCT and iatrogenic CTs were the most disease burdened groups. The highest need for advanced therapy and in-hospital mortality were observed for the acute myocardial infarction group, in contrast to PCT.

Conclusions: Within our cohort of patients, the invasive cardiac procedures overtake neoplastic causation of cardiac tamponade. The worst in-hospital prognosis was noted for CT following acute myocardial infarction and both iatrogenic invasive cardiac and cardiac surgery procedures. The highest long-term mortality was recorded for patients with end stage renal failure and the neoplastic group.
\end{abstract}

Key words: cardiac tamponade, neoplastic, iatrogenic, inflammatory, postpericardiotomy syndrome.

\section{Introduction}

Cardiac tamponade is a pericardial syndrome characterized by diastolic impairment due to the accumulation of pericardial fluid under pressure [1]. Clinical signs of cardiac tamponade (CT) include tachycardia, hypotension, pulsus paradoxus, raised jugular venous pressure, muffled heart sounds, and decreased electrocardiographic voltage with electrical alternans. The occurrence of hemodynamic abnormalities and clinical symptoms depends on the rate of fluid accumulation relative to pericardial stretch and the effectiveness of compensatory mechanisms [2]. CT may be an acute life-threatening condition (e.g. CT by hemopericardium), or may present as a subacute condition allowing in some cases delayed treatment. The causes of cardiac tamponade are various: iatrogenic factors, postpericardi- otomy syndrome, malignancies, uremia, heart rupture as complication in case of myocardial infarction, aortic dissection, hypothyroidism, idiopathic linked to inflammatory or immune processes, drug side effects etc. [1, 3, 4].

In a patient with clinical suspicion of cardiac tamponade, ECG and echocardiography are primary tools for CT diagnosis, and to a lesser extent computed tomography. The treatment of CT involves usually drainage of the pericardial fluid, preferably by needle pericardiocentesis (PCC) or a surgical approach [4].

\section{Aim}

Studies on the etiology of CT are scarce and usually include selected groups of patients. The aim of this study was to analyze all etiologies of $\mathrm{CT}$ in patients treated in the ter-

Address for correspondence: Mária Adamczyk MD, $3^{\text {rd }}$ Department of Cardiology, Faculty of Medical Sciences, Medical University of Silesia, Silesian Centre for Heart Diseases, 9 Marie Curie-Skłodowskiej St, 41-800 Zabrze, Poland, e-mail: maria.ester.adamczyk@gmail.com Received: 30.05.2021, accepted: 19.09.2021. 
tiary hospital with cardiology, cardiac surgery and intensive care departments. Additionally, for each group of main etiologies we determined the basic characteristics, in-hospital management, in-hospital and up to 2-year mortality.

\section{Material and methods}

The study was carried out in the Silesian Centre for Heart Diseases (SCCS) in Zabrze, Poland, which is tertiary clinical hospital having cardiac surgery, two cardiology and two intensive cardiology care departments. We analyzed retrospectively hospital databases and medical records of all patients older than 18 years with clinical and echocardiographic signs of cardiac tamponade who required pericardiocentesis or pericardiotomy treatment, between January 2008 and December 2018. Five patients with diagnosed $\mathrm{CT}$ requiring only conservative treatment as well as all patients whose medical history was not available or were lost to follow-up were excluded. For each of 340 enrolled patients with $\mathrm{CT}$, we established the final etiological diagnoses based on the clinical history, serum biomarkers, pericardial fluid culture, cytology and biochemistry analysis, results of computed tomography and magnetic resonance imaging (MRI) scans, as well as pericardial biopsy, if available. For each group with more than 5 patients, the baseline characteristics, length of hospital stay, in-hospital management and long term follow-up were analyzed.

latrogenic etiology was divided upon early onset (up to 48 hours after invasive cardiac (IC) or cardiac surgery procedure (iatrogenic cardiac surgery - ICS)) or late onset ( 7 days), which was considered as a separate postpericardiotomy syndrome group (PCT). The invasive cardiac procedures concerning iatrogenic CT performed in our center were: atrial fibrillation ablation, percutaneous coronary intervention, cardiovascular implantable electronic device, temporary pacing electrode, left atrial appendage closure, transcatheter aortic valve implantation and endomyocardial biopsy.

Neoplastic etiology of CT was assigned in case of neoplastic cells in pericardial fluid or presence of concomitant malignant disease. All patients with diagnosed CT caused by connective tissue diseases, infectious or non-infectious pericarditis were assigned to the inflammatory exudative pericarditis group. Uremic CT was established in patients with end stage renal failure in dialysis or predialysis in which other causes were excluded.

Data on long-term follow-up/mortality for all patients were obtained from the Silesian Cardiovascular Base formed as a result of an agreement between the $3^{\text {rd }}$ Department of Cardiology, Faculty of Medical Sciences in Zabrze, Medical University of Silesia, Katowice, the SCCS and the RegionalSilesian Branch of the National Health Fund in Katowice.

\section{Statistical analysis}

The distribution of continuous variables was tested using the Shapiro-Wilk test. None of the variables had a normal distribution. Continuous variables were presented in the form of median and interquartile range (IQR). Categori- cal variables were presented as percentages. In-hospital and post-discharge mortality was presented as the allcause crude death rate since hospital admission. Statistica 13.1 from TIBCO Inc. and Microsoft Excel were used for statistical and graphic data processing.

\section{Results}

Over an 11-year period 340 patients with CT were diagnosed and treated in our cardiac center, of whom 56\% were men. The total median hospital stay was 10 [14] days. Among particular etiologies 8 main subgroups were identified and 7 (beside OTHER group) were further analyzed: iatrogenic group after invasive cardiac procedures, iatrogenic after cardiac surgery procedures, postpericardiotomy syndrome group, neoplastic (NPL), inflammatory (INFL), uremic (UREMIC), acute myocardial infarction complication (AMI) group. In the group of OTHER $(n=13)$ the following etiologies were included: aortic dissection (4 patients), right sided heart failure with restriction (2 patients), postmyocardial infarction syndrome (1 patient), postradiation (1 patient), trauma with chest perforation (1 patient), coagulation abnormality (1 patient), unknown etiology (3 patients). The proportion of particular groups of etiologies are shown in Figure 1.

In our study cohort the highest median values of age 74 (20) and 70 (16.6) were found in AMI and IC groups respectively. The lowest median values of age 57-58 were reported in NPL, INFL and PCT groups. The longest hospital stay was typical for the ICS group, 25 (13) days, and 16 (17) days for the INFL group, in contrast to 6 (8) days for NPL and 6 (5) days in the PCT group. Women were in a majority in the NPL (66\%) and IC group (52\%). Median APTT ratio (coagulation time of test-to-reference plasma) values (normal range: $26-40 \mathrm{~s}$ ) were high in the UREMIC group, $42.1 \mathrm{~s}$ (12.6). High median prothrombic time and international normalized ratio were found in the PCT group

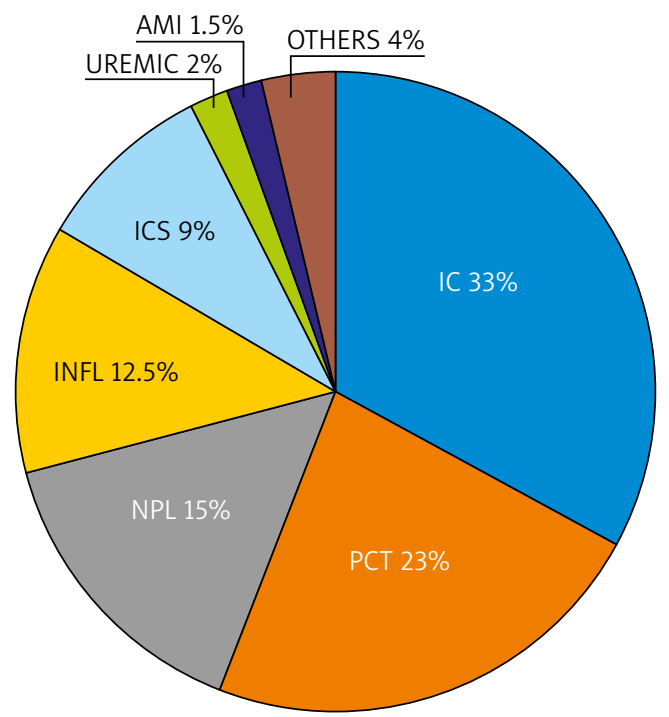

Figure 1. Particular groups of etiologies of 340 cardiac tamponades diagnosed and treated between January 2008 and December 2018 AMI - acute myocardial infarction complication, IC - iatrogenic after invasive cardiac procedures, ICS - iatrogenic after cardiac surgery procedures, $\mathrm{PCT}$ - postpericardiotomy syndrome, NPL - neoplastic, INFL - inflammatory. 
Baseline characteristics, management and long-term outcomes of different etiologies of cardiac tamponade evaluated in a cohort of 340 patients

Table I. Baseline characteristics, disease burden and laboratory parameters of patients with different etiologies of cardiac tamponade hospitalized between January 2008 and December 2018

\begin{tabular}{|c|c|c|c|c|c|c|c|}
\hline Parameter & $\begin{array}{c}\text { IC } \\
(n=112)\end{array}$ & $\begin{array}{c}\text { ICS } \\
(n=31)\end{array}$ & $\begin{array}{c}\text { PCT } \\
(n=78)\end{array}$ & $\begin{array}{c}\text { NPL } \\
(n=50)\end{array}$ & $\begin{array}{c}\text { INFL } \\
(n=43)\end{array}$ & $\begin{array}{l}\text { UREMIC } \\
(n=8)\end{array}$ & $\begin{array}{c}\text { AMI } \\
(n=5)\end{array}$ \\
\hline Age [years] & $70(16.6)$ & $64(15)$ & $58(15.7)$ & $57(13.4)$ & $57.5(26)$ & $65(17)$ & $74(20)$ \\
\hline Hospital stay [days] & $13(15)$ & $25(13)$ & $6(5)$ & $6(8)$ & $16(17)$ & $11(12)$ & $14(29)$ \\
\hline (\%) of men & 48 & 55 & 74 & 34 & 53 & 75 & 80 \\
\hline Prior CAD (\%) & 44 & 29 & 33 & 8 & 23 & 37.5 & 20 \\
\hline Prior AF (\%) & 38 & 39 & 24 & 18 & 25,5 & 62.5 & 0 \\
\hline Prior CHF (\%) & 24 & 42 & 55 & 8 & 23 & 50 & 0 \\
\hline Prior AMI (\%) & 27 & 16 & 11.5 & 4 & 7 & 12.5 & 40 \\
\hline Prior PAD (\%) & 12.5 & 29 & 17 & 4 & 9 & 37.5 & 40 \\
\hline Prior CKD (\%) & 12.5 & 10 & 13 & 6 & 14 & 100 & 20 \\
\hline Prior AH (\%) & 50 & 48 & 64 & 28 & 28 & 50 & 80 \\
\hline Prior DM (\%) & 20 & 13 & 23 & 12 & 12 & 37.5 & 20 \\
\hline Obesity (\%) & 7 & 13 & 33 & 2 & 2.3 & 0 & 0 \\
\hline APTT & $35.95(13.3)$ & $38.1(15.3)$ & $40.2(13.7)$ & $34.3(10.85)$ & $36.95(8.1)$ & $42.1(12.6)$ & $71.5(64.7)$ \\
\hline PT & $13.5(2.1)$ & $13.8(2.7)$ & $22.5(13)$ & $14.65(2.9)$ & $15.05(2.5)$ & $16.7(3.3)$ & $14.0(2.2)$ \\
\hline INR & $1.06(0.19)$ & $1.08(0.257)$ & $1.99(1.36)$ & $1.2(0.26)$ & $1.2(0.26)$ & $1.37(0.37)$ & $1.1(0.19)$ \\
\hline GFR & $60.0(38)$ & $82.9(50.34)$ & $60.0(35.7)$ & $60.0(52.15)$ & $65.5(48.4)$ & 30.5 (11) & $35.9(10)$ \\
\hline CREAT [mmol/l] & $87.4(43.7)$ & $82.58(37.5)$ & $95.2(50.9)$ & $83.3(81)$ & $83.0(39.6)$ & $203.6(106.9)$ & $141.0(62.5)$ \\
\hline $\mathrm{PLT}\left[\times 10^{9} / \mathrm{I}\right]$ & $203(84)$ & $208(105)$ & $343(262)$ & $257.5(150)$ & $240(165)$ & 261.5 (191) & $181(69)$ \\
\hline $\mathrm{HCT}$ & $0.397(0.07)$ & $0.37(0.08)$ & $0.32(0.08)$ & $0.36(0.09)$ & $0.36(0.1)$ & $0.38(0.06)$ & $0.39(0.03)$ \\
\hline $\mathrm{HGB}[\mathrm{mmol} / \mathrm{l}]$ & $8.4(1.7)$ & $7.8(2.1)$ & $6.40(1.8)$ & $7.5(2.0)$ & 7.65 (2.4) & $7.7(1.5)$ & $8.8(1.2)$ \\
\hline $\mathrm{RBC}\left[\times 10^{12} / \mathrm{I}\right]$ & $4.4(0.78)$ & $4.08(0.86)$ & $3.575(0.99)$ & $3.92(0.9)$ & $4.14(1.07)$ & $4.09(1.35)$ & $4.5(0, .32)$ \\
\hline $\mathrm{WBC}\left[\times 10^{9} / 1\right]$ & 7.38 (3.5) & $7.86(3.9)$ & $10.32(4.82)$ & $10.51(7.27)$ & $8.07(5.4)$ & $7.23(6.05)$ & $14.4(1.7)$ \\
\hline
\end{tabular}

$\mathrm{AMI}$ - acute myocardial infarction, NPL - neoplasm, IC - iatrogenic after invasive cardiac procedures, ICS - iatrogenic after cardiosurgery procedures,

$\mathrm{PCT}$ - postpericardiotomy syndrome, AF - atrial fibrillation, APTT - activated partial thromboplastin time, CAD - coronary artery disease, CHF - chronic heart failure,

CKD - chronic kidney disease, CS - cardiac surgery, GFR - glomerular filtration rate, HCT - hematocrit, HGB hemoglobin, INR - international normalized ratio,

CREAT - creatinine, MI - myocardial infarction, PAD - peripheral artery disease, PLT - platelets, PT - prothrombin time, RBC - red blood cells, WBC - white blood cells.

(22.5 s and 1.99 respectively). At hospital admission, anemia was present in PCT patients and the sign of renal impairment renal impairment with the lowest glomerular filtration rate (GFR) was especially found in UREMIC and AMI groups. The AMI patients were additionally burdened with leukocytosis. The UREMIC, PCT and both IC and ICS groups were the most disease burdened groups of patients. All baseline characteristics, laboratory parameters and data on comorbidities are summarized in Table I.

The majority of pericardial fluid drainages were performed by pericardiocentesis (PCC); however, $52 \%$ of patients from the ICS group underwent PCC and $48 \%$ of them required surgery (Table II). Three of 50 patients and 3 of 43 patients from NPL and INFL groups respectively required fenestration of the pericardial sac. The highest total volume of pericardial fluid was drained after AMI (median of 1500 (1900) ml), UREMIC (1140 (500) $\mathrm{ml}$ ) and NPL (910 (710) ml) etiologies of CT. The lowest volumes of fluid were drained in the IC group (median of $390(460) \mathrm{ml}$ ).

Inotropes together with mechanical ventilation support were used in a high percentage in AMI patients (80\%, 60\% respectively), then in ICS (29\%, $13 \%$ respectively) and IC patients (28\%, 15\% respectively). The lowest frequency of such treatment was required in the PCT group. The type of treatment and in-hospital management data for all etiologies are summarized in Table II. In-hospital, annual and 2-year mortality rates for different etiology groups are presented in Figure 2.

\section{Discussion}

We present the largest contemporary study on CT assessing 340 consecutive patients diagnosed and treated for CT over an 11-year period. Before the era of percutaneous invasive cardiac procedures, malignancy (primarily lung and breast cancer) was the leading cause of CT [5, 6]. Our series from a tertiary care cardiology hospital confirms the iatrogenic cause as the predominant one (42\%) and our observations are in line with a recent study by Orbach et al. [7].

latrogenic $\mathrm{CT}$ is a rare but life-threatening complication [8]. The risk of iatrogenic CT or pericardial effusion increases with the need for transseptal puncture and intraprocedural anticoagulation [9]. In our study, among 340 included patients, 112 (33\%) after IC procedures and 31 (9\%) after ICS procedures were identified. In the group of IC the lowest total pericardial volume was drained. It shows that iatrogenic CT is more likely to develop rapidly with coexistence of relatively small pericardial hemorrhages. In $82 \%$ of IC patients PCC was used as a successful emergency treatment, 
Table II. In-hospital management of patients with different etiologies of cardiac tamponade hospitalized between January 2008 and December 2018

\begin{tabular}{lccccccc} 
Parameter & $\begin{array}{c}\text { IC } \\
(n=112)\end{array}$ & $\begin{array}{c}\text { ICS } \\
(n=31)\end{array}$ & $\begin{array}{c}\text { PCT } \\
(n=78)\end{array}$ & $\begin{array}{c}\text { NPL } \\
(n=50)\end{array}$ & $\begin{array}{c}\text { INFL } \\
(n=43)\end{array}$ & $\begin{array}{c}\text { UREMIC } \\
(n=8)\end{array}$ & $\begin{array}{c}\text { AMI } \\
(n=5)\end{array}$ \\
PCC, $n(\%)$ & $92(82 \%)$ & $17(52 \%)$ & $47(60 \%)$ & $49(98 \%)$ & $40(93 \%)$ & $7(87.5 \%)$ & $3(60 \%)$ \\
\hline Pericardiotomy, $n(\%)$ & $16(14 \%)$ & $12(42 \%)$ & $31(40 \%)$ & 0 & $2(5 \%)$ & $1(12.5 \%)$ & $1(20 \%)$ \\
\hline PCC + pericardiotomy, $n(\%)$ & $4(4 \%)$ & $2(6 \%)$ & 0 & $1(2 \%)$ & $1(2 \%)$ & 0 & $1(20 \%)$ \\
\hline Fenestration, $n$ of patients & 1 & 1 & 2 & 3 & 3 & 0 & 0 \\
\hline Inotropes (\%) & 28 & 29 & 3 & 6 & 12 & 25 & 80 \\
\hline MVS (\%) & 15 & 13 & 1 & 6 & 9 & 12.5 & 60 \\
\hline Data available in $\times(\%)$ of patients & $76 \%$ & $15 \%$ & $74 \%$ & $90 \%$ & $74 \%$ & $50 \%$ & $60 \%$ \\
\hline Drained pericardial fluid - first access $[\mathrm{ml}]$ & $320(360)$ & $520(180)$ & $650(440)$ & $655(510)$ & $575(495)$ & $860(900)$ & $550(350)$ \\
\hline Drained pericardial fluid - total $[\mathrm{ml}]$ & $390(460)$ & $600(465)$ & $700(400)$ & $910(710)$ & $630(860)$ & $1140(500)$ & $1500(1900)$ \\
\hline
\end{tabular}

AMI - acute myocardial infarction complication, IC - iatrogenic after invasive cardiac procedures, ICS - iatrogenic after cardiosurgery procedures, MVS - mechanical ventilation support, NPL - neoplasm, PCT - postpericardiotomy syndrome, inotropes - sympathomimetic amine therapy, PCC - pericardiocentesis.

and $18 \%$ required thoracotomy. After the $\mathrm{AMI}$ group, IC as well as ICS patients had the highest need for advanced treatment and higher in-hospital mortality among all studied patients. However, overall mortality up to 2 years was definitely superior in the ICS group in comparison to CTS after IC procedures (Figure 2). Additionally, ICS patients had the longest hospital stay among all analyzed groups (median: 25 days) and $42 \%$ required reoperation. The data on drained volumes are not satisfactory in the ICS group, mostly because of the urgent need for surgical decompression and the lack of such information in patients' histories.

The study results concerning CT after defined percutaneous invasive cardiac procedures performed in our clinical center between 2006 and 2018 have been recently published [10].

The postpericardiotomy syndrome occurs mostly 1 to 6 weeks after cardiac surgery, but clinical symptoms may occur as late as several weeks to months after surgery and may be associated with significant morbidity. It is believed that PCT results from a heightened immune response to injury following cardiothoracic surgery $[11,12]$. Incidence of PCT is close to $10 \%$ but varies among studies from $2 \%$ to $30 \%$, as well as according to type of cardiac surgery performed [13]. Although most patients have an uncomplicated clinical course with good prognosis after standard pharmacological treatment, some studies report cases of patients with late diagnosis that required pericardial drainage or even redo surgery [11]. In our study cohort, PCT syndrome was the second most frequent etiology of the CT (78 of 340), although with the shortest hospital stay, the lowest need for advanced therapy and with the best prognosis.

In our 340 consecutive patients 50 (15\%) of them were assigned to the NPL group. Cardiac tamponade in case of the NPL process was present in 50/96 (52\%) in a study by Navarette et al. [5], 74/114 (65\%) by Cornily et al. [14] and 43/136 (32\%) by Sánchez-Enrique et al. [6]. In-hospital stay for the NPL group was short, mostly because of patients being referred to our center for diagnostic and therapeutic PCC. In only $2 \%$ of them pericardiotomy was additionally performed as PCC was not satisfactory. Only $6 \%$ of them required advanced treatment. Although the in-hospital mortality was not high, the annual and 2-year mortality

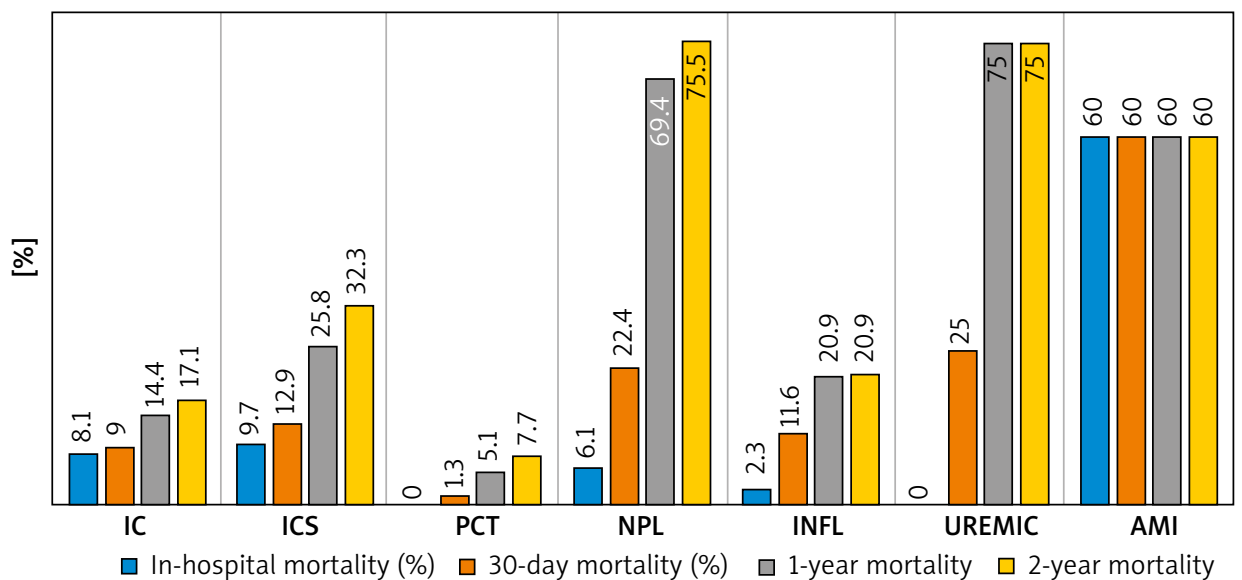

Figure 2. In-hospital, 30-day, 1-year and 2-year mortality for different etiology groups of patients with cardiac tamponade, hospitalized between January 2008 and December 2018. In-hospital and post-discharge mortality is presented as all-cause crude death rate since hospital admission. For that reason, 30-day, 1-year and 2-year mortality in AMI group remained high

AMI - acute myocardial infarction, CT - cardiac tamponade, NPL - neoplasm, IC - iatrogenic after invasive cardiac procedures, ICS - iatrogenic after cardiac surgery procedures, INFL - inflammatory, PCT - postpericardiotomy syndrome. 
was significant, reaching 2-year mortality of up to $75 \%$. Nevertheless, such high mortality may be consequence of primary disease, not CT alone.

In our analysis, all causes, infectious or noninfectious, as well as collagen disease inflammatory exudative pericarditis, were united together in the INFL group. The purpose was to define a bigger group to obtain reliable statistical results. The INFL group consisted of 43 (12.5\%) patients, 6 with a diagnosis of viral, 6 of bacterial (2 tuberculosis), 6 of collagen disease, 1 toxoplasmosis and 25 of unknown inflammatory etiology. Most previous publications focused on individual and different inflammatory etiologies, so the nomenclature, in our opinion, is not consistent. INFL was one of the youngest groups, with mean age of 57.5 (26), with the second longest median hospital stay. Concerning in-hospital management and long-term mortality, this group was in the middle position among all groups.

Uremic pericarditis typically occurs in patients with end-stage renal disease [15]. Cardiac tamponade, a further complication of uremic pericarditis, is more common in dialyzed than in non-dialyzed patients with chronic renal failure, and must be in most cases treated by pericardiocentesis [16]. Some studies defined the number of uremic CTs as 12 of 96 patients (12.5\%) [5] or 11/50 (20\%) [17]. Our UREMIC group consisted of 8 patients with end-stage renal disease, of whom $70 \%$ were men. It was the group of patients with the highest percent of co-morbidities, and although in-hospital mortality was 0\%, annual and 2-year mortality was equal to the NPL group. Such high mortality may be a consequence of primary disease, not CT alone.

Cardiac tamponade during myocardial infarction is mainly due to heart rupture, or more rarely to hemorrhagic evolution of post-myocardial infarction pericarditis [1]. In our analysis there were only 8 patients with $\mathrm{CT}$ as a complication of myocardial infarction. Such a small number of wall perforations during AMI is most likely due to the implementation of more precise diagnostic methods and more effective treatments, including invasive interventions [18]. It was group with the highest total volume of drained pericardial fluid, the highest percentage of required intensive care therapy ( $80 \%$ of patients with inotropes and $60 \%$ with mechanical ventilatory support) and the highest in-hospital mortality of about $60 \%$. It may be obviously explained mostly by rupture of the heart and massive hemorrhage.

\section{Conclusions}

Most CTs are observed after invasive cardiac procedures, following by postpericardiotomy syndrome, neoplasm, cardiac surgery procedures, inflammatory, uremic and acute myocardial infarction complications. Pericardiocentesis was used as successful first-line emergency treatment in the majority of cases. The worst in-hospital prognosis was noted for CT following acute myocardial infarction complications and both invasive cardiac and cardiac surgery iatrogenic CTs. The highest annual and 2-year mortality was recorded for UREMIC and NPL groups, which is most likely the result of the underlying disease.

\section{Disclosure}

The authors report no conflict of interest.

\section{References}

1. Imazio M. Ten questions regarding cardiac tamponade. G Ital Cardiol 2018; 19: 471-478.

2. Spodick DH. Acute cardiac tamponade. N Engl J Med 2003; 349: 684-690.

3. Imazio M, De Ferrari GM. Cardiac tamponade: an educational review. Eur Heart J Acute Cardiovasc Care 2021; 10: 102-109.

4. Adler Y, Charron P, Imazio M, Badano L, Barón-Esquivias G, Bogaert J, Brucato A, Gueret P, Klingel K, Lionis C, Maisch B, Mayosi B, Pavie A, Ristić AD, Sabaté Tenas M, Seferovic P, Swedberg K, Tomkowski W, Achenbach S, Agewall S, AlAttar N, Angel Ferrer J, Arad M, Asteggiano R, Bueno H, Caforio ALP, Carerj S, Ceconi C, Evangelista A, Flachskampf F, Giannakoulas G, Gielen S, Habib G, Kolh P, Lambrinou E, Lancellotti P, Lazaros G, Linhart A, Meurin P, Nieman K, Piepoli MF, Price S, Roos-Hesselink J, Roubille F, Ruschitzka F, Sagristà Sauleda J, Sousa-Uva M, Uwe Voigt J, Luis Zamorano J, Zamorano JL, Aboyans V, Achenbach S, Agewall S, Badimon L, Barón-Esquivias G, Baumgartner H, Bax JJ, Bueno H, Carerj S, Dean V, Erol Ç, Fitzimons D, Gaemperli O, Kirchhof P, Kolh P, Lancellotti P, Lip GY, Nihoyannopoulos P, Piepoli MF, Ponikowski P, Roffi M, Torbicki A, Vaz Carneiro A, Windecker S, Shuka N, Sisakian H, Mascherbauer J, Isayev E, Shumavets V, Van Camp G, Gatzov P, Hanzevacki JS, Moustra HH, Linhart A, Møller JE, Aboleineen MW, Põder P, Lehtonen J, Antov S, Damy T, Schieffer B, Dimitriadis K, Kiss RG, Rafnsson A, Arad M, Novo S, Mirrakhimov E, Stradinš P, et al. 2015 ESC Guidelines for the diagnosis and management of pericardial diseases. Eur Heart J 2015; 36: 2921-2964.

5. Navarrete CO, Marín Ortuńo F, Pineda Rocamora J, Luján Martínez J, García Fernández A, Climent Payá VE, Martínez Martínez JG, Aranda López I, Sogorb Garri F. Debemos pensar en una etiología específica en pacientes con taponamiento cardíaco? Rev Esp Cardiol 2002; 55: 493-498.

6. Sánchez-Enrique C, Nuńez-Gil IJ, Viana-Tejedor A, De Agustín A, Vivas D, Palacios-Rubio J, Vilchez JP, Cecconi A, Macaya C, Fernández-Ortiz A. Cause and long-term outcome of cardiac tamponade. Am J Cardiol 2016; 117: 664-669.

7. Orbach A, Schliamser JE, Flugelman MY, Zafrir B. Contemporary evaluation of the causes of cardiac tamponade: acute and long-term outcomes. Cardiol J 2016; 23: 57-63.

8. Adamczyk M, Wasilewski J, Niedziela J, Rozentryt P, Gąsior M. Pericardial tamponade as a complication of invasive cardiac procedures: a review of the literature. Adv Interv Cardiol 2019; 15: 394-403.

9. De Ponti R, Cappato R, Curnis A, Della Bella P, Padeletti L, Raviele A, Santini M, Salerno-Uriarte JA. Trans-septal catheterization in the electrophysiology laboratory: data from a multicenter survey spanning 12 years. J Am Coll Cardiol 2006; 47: 1037-1042.

10. Adamczyk M, Niedziela JT, Wasilewski J, Zembala MO, Kalarus Z, Gąsior M. Prevalence, management and outcomes of cardiac tamponade complicating 66,812 invasive cardiac procedures: single-center clinical registry. Adv Interv Cardiol [Internet]. 2021 [cited 2021 Dec 15];17(64):193-9. Available from: https://doi.org/10.5114/aic.2021.107499.

11. Imazio M, Hoit BD. Review: post-cardiac injury syndromes. An emerging cause of pericardial diseases. Int J Cardiol 2013; 168: 648-652.

12. Imazio M, Brucato A, Rovere ME, Gandino A, Cemin R, Ferrua S, Maestroni S, Barosi A, Simon C, Ferrazzi P, Belli R, Trinchero R, Spodick D, Adler Y. Contemporary features, risk factors, and prognosis of the post-pericardiotomy syndrome. Am J Cardiol 2011; 108: 1183-1187.

13. Miller RH, Horneffer PJ, Gardner TJ, Rykiel MF, Pearson TA. The epidemiology of the postpericardiotomy syndrome: a common complication of cardiac surgery. Am Heart J 1988; 116: 1323-1329.

14. Cornily JC, Pennec PY, Castellant P, Bezon E, Le Gal G, Gilard M, Jobic Y, Boschat J, Blanc JJ. Cardiac tamponade in medical patients: a 10-year follow-up survey. Cardiology 2008; 111: 197-201.

15. Nesheiwat Z, Lee JJ. Uremic Pericarditis. StatPearls Publishing; 2021. Accessed May 13, 2021. http://www.ncbi.nlm.nih.gov/pubmed/30725605.

16. Koopot R, Zerefos NS, Lavender AR, Pifarré R. Cardiac tamponade in uremic pericarditis. Surgical approach and management. Am J Cardiol 1973; 32: 846-849.

17. Kabukcu M, Demircioglu F, Yanik E, Basarici I, Ersel F. Pericardial tamponade and large pericardial effusions: causal factors and efficacy of percutaneous catheter drainage in 50 patients. Texas Hear Inst J 2004; 31: 398-403.

18. Gasior M, Pres D, Wojakowski W, Buszman P, Kalarus Z, Hawranek M, Gierlotka M, Lekston A, Mizia-Stec K, Zembala M, Poloński L, Tendera M. Causes of hospitalization and prognosis in patients with cardiovascular diseases Secular trends in the years 2006-2014 according to the SILesian CARDiovascular (SILCARD) database. Pol Arch Med Wewn 2016; 126: 754-762. 